J. Clin. Chem. Clin. Biochem.

Vol. 14, 1976, pp. 27-30

\title{
Ein Verfahren zur enzymkinetischen Bestimmung von Glucose
}

\author{
Von $H$. Keller, $\boldsymbol{V}$. Wolf \\ Institut für Klinische Chemie und Hämatologie St. Gallen,
}

U. Faust, W. Bleicher und J. Becker

Institut für Biomedizinische Technik Stuttgart

(Eingegangen am 23. Juni/28. August 1975)

Zusammenfassung: Es wird ein reaktionskinetisches Verfahren zur Bestimmung der Glucose in biologischen Flüssigkeiten beschrieben. Dabei wird mittels Glucosedehydrogenase Glucose zu Gluconsäure oxydiert und NAD zu NADH reduziert. Die Bildungsgeschwindigkeit des NADH wird in einem LKB Reaction-Rate-Meter verfolgt. Neben der Analogdarstellung wird die Reaktionsgeschwindigkeit mit einem neu entwickelten Kinetikrechner bestimmt und die Spezifikationen des Rechners werden beschrieben.

Vorzug des neuen Verfahrens ist die hohe Durchsatzgeschwindigkeit und gute Reproduzierbarkeit der Resultate.

\section{Enzyme kinetic determination of glucose}

Summary: A reaction kinetic method is described for the determination of glucose in biological fluids. Glucose is oxidized to gluconic acid and NAD is reduced to NADH in the presence of glucose dehydrogenase. The rate of formation of NADH is followed in a LKB Reaction-Rate-Meter. Together with the analogue read-out, the rate of reaction is determined with a newly developed kinetic calculator. The specifications of the calculator are given.

The new method has the advantage of a high rate of throughput and good reproducibility.

\section{Einfuhrung}

Zur Bestimmung der Glucose in Blut, Plasma, Serum oder Urin wird heute allgemein den enzymatischen Verfahren der Vorzug gegeben, da die Spezifität aller anderen Methoden eindeutig geringer ist. Am häufigsten dürfte in den europäischen Laboratorien das Glucoseoxidase/Peroxidase-Verfahren ${ }^{1}$ ) (1) eingesetzt werden. Fehler dieses in vielen Modifikationen (2) beschriebenen, zweistufigen Verfahrens ist die geringe Spezifität und die Störanfälligkeịt der zweiten Stufe, der Peroxidase-Reaktion. Úm sie zu umgehen wurde der $\mathrm{O}_{2}$-Verbrauch der Reaktion mit Hilfe einer Clärk-Elektrode verfolgt und daraus die Glucosekonzentration berechnet (3). Aber auch dieșes Verfahren ist de fakto zweistufig: Da im Untersuchungsmaterial in der Regel Katalase vorhanden ist, durch die der Sauerstoff wieder freigesetzt wird, muß er durch geeignete Hilfsreaktionen

1) Enzyme: Glucoseoxidase EC 1.1.3.4

Glucosedehydrogenase EC 1.1.1.118

Peroxidase EC 1.11.1.7

Hexokinase EC 2.7.1.1

Glucose-6-phosphat-dehydrogenase EC 1.1.1.49

Katalase EC 1.11.1.6 verbraucht werden, z. B. durch Oxydation von Jodid in Gegenwart von Ammoniummolybdat.

Als Referenzmethode ist die Kombination der unspezifischen Hexokinase mit der spezifischen Glucose-6phosphat-dehydrogenase von Bücher et al (4) beschrieben worden. Dieses Verfahren ist spezifisch, benötigt nicht zwingend einen Deproteinisierungsschritt und ist rascher durchfuihrbar, als die Glucoseoxidase/PeroxidaseReaktion. Die Berechnung kann nach dem Absolutprinzip mit Hilfe des Extinktionskoeffizienten ausgefuhrt werden.

Grundsätzlich kann das Glucoseoxidase/Peroxidasewie auch das Hexokinaseverfahren eingesetzt werden, um die Glucose auf kinetischem Wege zu bestimmen $(5,6)$.

Die Tatsache, daß es sich bei diesen Verfahren um gekoppelte żwèistufige Reaktionen handelt, macht eine kinetische Bestimmung jedoch problematisch. Bei Bestimmungen dieser Art muß davon ausgegangen werden, daß die Zahl der aktiven Stellen der Enzymmoleküle die der Substratmoleküle übersteigt, so daß diese gescḥwịndigkeitșbestimmend sind (7). Ein (teilweiser) 
Aktivitätsverlust der beteiligten Enzyme macht das Verfahren rasch unbrauchbar. Deshalb ist ein einstufiges Verfahren bei enzymkinetischen Substratmessungen einem zweistufigen vorzuziehen.

Vor kurzem (8) wurde auf die Einsatzmöglichkeit der Glucosedehydrogenase zur „Blutzuckerbestimmung" hingewiesen. Es war deshalb naheliegend, dieses Enzym auch zur enzymkinetischen Glucosebestimmung heranzuziehen.

\section{Material und Methoden}

\section{Reagenzien}

1. Deproteinisierungsreagenz: $3 \mathrm{~g}$ Perchlorsäure gelöst in $100 \mathrm{ml}$ dest. Wasser

2. Phosphatpuffer: $0,12 \mathrm{~mol} / 1 \mathrm{pH} 7,6$, enthaltend $0,15 \mathrm{~mol} / \mathrm{l}$ Natriumchlorid.

3. Enzym/NAD Lösung: 1760 IU Glucosedehydrogenase (E. Merck, Darmstadt) und $268 \mathrm{mg}$ NAD (entsprechend $0,405 \mathrm{mmol}$ ) werden in $50 \mathrm{ml}$ Phosphatpuffer gelöst, ,Startreagenz".

4. Primärstandards: Durch Einwaage und Verdünnung wurden folgende Glucosekonzentrationen hergestellt:

$\begin{array}{lllllllll}25 & 50 & 100 & 200 & 300 & 400 & 500 & \mathrm{mg} / \mathrm{dl}\end{array}$

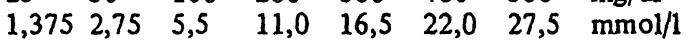

5. Kontrollseren:

Labtrol LT-42 P, Deklaration (Glucoseoxidase/Peridmethode): $108(100 \mathrm{bis} 116) \mathrm{mg} / \mathrm{dl}$ $5,94(5,5-6,38) \mathrm{mmol} / 1$

Pathotrol-PT-66H, Deklaration (Glucoseoxidase-Peridmethode) $275(255-395) \mathrm{mg} / \mathrm{dl}$

$15,125(14,025-16,225) \mathrm{mmol} / \mathrm{l}$

Qualtrol QT-29b, Erfahrungswert (Glucoseoxidase-Peridmethode) $105(96-114) \mathrm{mg} / \mathrm{dl}$

$$
5,775(5,28-6,27) \mathrm{mmol} / \mathrm{l}
$$

Instrumentation

Als Meßinstrument wurde der LKB 8600 Reaction Rate Analyzer eingesetzt. Zur Auswertung und Steuerung wurde ein Zusatzgerät zum LKB-System entwickelt. Das Blockschaltbild dieses Kinetikrechners ist in der Abbildung 1 wiedergegeben. Am LKB-Photometer 8600 wird der ,Timer" durch einen internen Eingriff reversibel außer Betrieb gesetzt. Nach dem manuellen Start des Photometers läuft der Meßzyklus $\mathrm{ab}$ und wird so oft wiederholt, bis der Vorrat an Probengefäßen erschöpft ist. Vom Photometer wird ein Signal bereitgestellt,

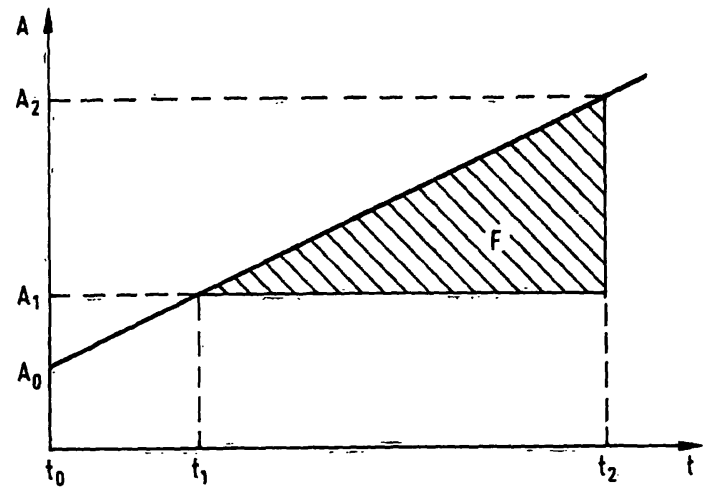

Abb. 1b. Prüfung des Reaktionsverlaufes auf seine Linearität Ergebnis der Zweipunktmessung: $v=\frac{A_{2}-A_{1}}{t_{2}-t_{1}}$
Meßergebnis durch Integration: $\bar{v}=\frac{2}{\left(t_{2}-t_{1}\right)^{2}} \cdot F$ $F=\int_{t_{1}}^{t_{2}}\left[A(t)=A_{1}(t)\right] d t$

bei linearem Verlauf ist:

$F=\frac{1}{2}\left(A_{2}-A_{1}\right)\left(t_{2}-t_{1}\right)$ dann wird: $v=\bar{v}$

welches den Zeitpunkt $\left(t_{0}\right)$ angibt, an dem die Küvette sich in Meßposition befindet (Abb. $1 \mathrm{~b}$ ). Dieses Signal startet einen Zeitgeber im Kinetikrechner, mit dem eine variable Vorlaufzeit bis zum Beginn der Messung eingeschaltet wird. Am Ende der Vorlaufzeit $t_{1}$ beginnt die Absorptionsmessung (Zeitkonstante etwa $100 \mathrm{~ms}$ ), das analoge Resultat wird gespeichert. Gleichzeitig wird ein zweiter Zeitgeber gestartet, welcher die Zeit $t_{2}$ bestimmt, an der die Messung beendet werden soll. Am Ende des Meßzyklus wird außerdem ein Steuerbefehl an das Photometer gegeben, womit der Probenwechsel eingeleitet wird. Die Absorptionsdifferenz an den beideñ Meßpunkten wird auf die Meßzeit bezogen (v) mit einem variablen Eichfaktor multipliziert und nach analog/digital-Wandlung über einen Zahlendrucker ausgegeben. Zur Kontrolle des Reaktionsverlaufes auf Linearität wird das Absorptionssignal im Meßintervall außerdem integriert und mit einem von der Meßzeit abhängigen Faktor multipliziert. Das Ergebnis $\bar{v}$ wird mit dem durch die Z Zwei-Punkt-Messung gewonnenen Wert v verglichen. Bei linearem Reaktionsverlauf stimmen beide überein. Überschreitet die Abweichung eine vorgegebene Schwelle, so wird das Meßergebnis mit einem Fehlerkennzeichen $(L)$ versehen. Ist die Probe dunkler, bzw. heller als ein vorgegebener

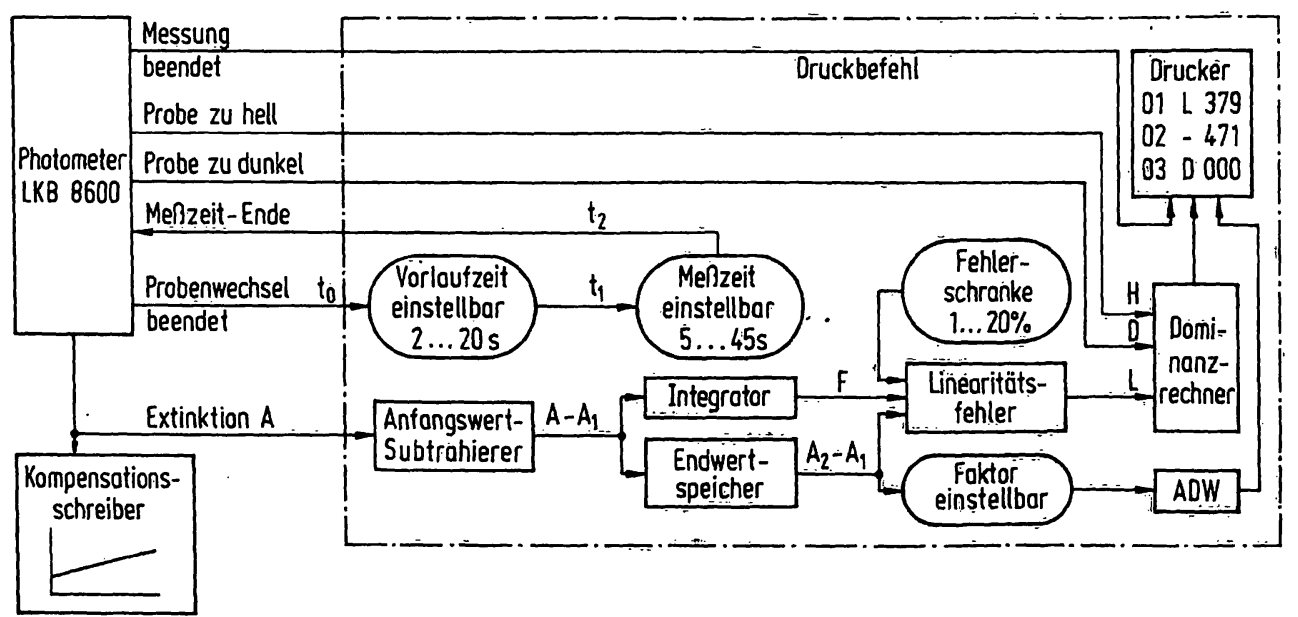

Abb. 1 a. Blockschaltbild des Kinetikrechners Einzelheiten s. Text 
Schwellenwert, gibt das Photometer kein Ausgangssignal ab. Der Drucker kennzeichnet diese Situation durch die Buchstaben D (dunkel) bzw. H (hell).

Durchführung des Bestimmungsverfahrens

In Vorversuchen war die optimale Versuchsanordnung ermittelt worden:

1. Blutentnahme:

$100 \mu \mathrm{l}$ Blut wurden mittels einer Kapillarpipette in $1 \mathrm{ml}$ Perchlorsäurelösung überführt und abzentrifugiert.

2. Beschickung der Reaktionsküvetten:

Vom Uberstand wurden $50 \mu 1$ in $1 \mathrm{ml}$ Phosphatpuffer überführt. Der Puffer befand sich in den Einmalküvetten des LKB-Systems, die vorgefülten Küvettenmagazine sind in einem Inkubator auf $35^{\circ} \mathrm{C}$ vorgewärmt. Wenn än Stelle von Blut Primär- oder Sekundärstandards verwendet wurden, so war das Vorgehen analog.

3. Durchführung der kinetischen Substratbestimmung: Gestartet wurde mit $200 \mu l$ Startreagenz (entsprechend 7,04 IU Enzym pro Einsatz). Nach der geräteinternen Mischung wurde durch den Rechner eine Vorlaufzeit von $5 \mathrm{~s}$ eingeschoben (während der keine Registrierung erfolgte) und danach während 5 s registriert. Anschließend Abbruch der Messung, Probenwechsel und erneuter Start. Der Vollausschlag des Schreibers betrug $0,2 \mathrm{E}$.

4. Vergleichsmethode:

Verglichen wurde mit der Glucoseoxidase-Peridmethode (Boehringer, Mannheim, Best. Nr. 15756).

5. Vergleichsuntersuchungen:

Von 200 Patienten wurde zweimal innert weniger Minuten Kapillarblut entnommen und die erste Probe für die Glucoseoxidase-Peridbestimmung, die zweite für die GlucosedehydrogenaseBestimmung eingesetzt.

\section{Ergebnisse}

\section{Linearitätsbereich und Präzision beim Einsatz von Primärstandards}

Wie Tabelle 1 zeigt, ist über den geprüften Konzentrationsbereich von $25-500 \mathrm{mg} / 100 \mathrm{ml}$ Glucose $(1,375-$ $27,5 \mathrm{mmol} / \mathrm{l}$ ) eine befriedigend lineare Beziehung zwischen der Glucosekonzenträtion und der Reaktionsgeschwindigkeit gegeben. Es ist bemerkenswert, daß bis einschließlich $400 \mathrm{mg} / 100 \mathrm{ml},(22 \mathrm{mmol} / \mathrm{l})$ die Standardabweichung nahezu gleich bleibt, erst bei $500 \mathrm{mg} /$ $100 \mathrm{ml},(27,5 \mathrm{mmol} / \mathrm{l})$ ist eine deutliche Zunahme erkennbar. Welchen Einfluß Vorlauf und Enzymkonzentration auf die Präzision haben, ist aus der Tabelle 2 ersichtlich. In Tabelle $2 \mathrm{a}$ sind die Variationen von Vorlauf und Enzymkonzentrationen wiedergegeben, in Tabelle $2 b$ die Auswirkungen auf den Variationskoeffizienten. Es ist offensichtlich, daß ein Vorlauf von $5 \mathrm{~s}$ nicht wesentlich unterschritten werden darf, wenn man nicht im Bereich der niedrigen Konzentrationen eine erhebliche Streuung in Kauf nehmen will. Andererseits bringt ein längerer Vorlauf keine Präzisionsverbesserung, wenn zugleich die Enzymmenge auf die Hälfte vermindert wird.

Tab. 2. Auswirkung von Variation des Vorlaufs und der eingesetzten Enzymaktivität (2a) auf den Variationskoeffizienten bei ansteigenden Konzentrationen von Primärstandards. (2b)

Tab. 2a

\begin{tabular}{llll}
\hline & $\begin{array}{l}\text { Vorlauf } \\
\text { [s] }\end{array}$ & $\begin{array}{l}\text { Meßzeit } \\
\text { [s] }\end{array}$ & $\begin{array}{l}\text { Enzym/Ansatz } \\
\text { [I. U.] }\end{array}$ \\
\hline A & 10 & 5 & 3,52 \\
B & 2 & 5 & 7,04 \\
C & 5 & 5 & 7,04 \\
\hline
\end{tabular}

Tab. $2 b$

\begin{tabular}{lcccc}
\hline $\begin{array}{l}\text { Glucose } \\
\text { [mmol/1] }\end{array}$ & [mg/dl] & \multicolumn{4}{c}{ Variationskoeffizient } \\
& & A\%] & B & C \\
\hline 1,375 & 25 & 38,5 & 10,5 & 6,0 \\
2,75 & 50 & 9,2 & 4,6 & 3,5 \\
5,5 & 100 & 3,2 & 2,6 & 2,1 \\
11,0 & 200 & 2,1 & 3,1 & 0,5 \\
22,0 & 400 & 1,1 & 3,1 & 1,5 \\
\hline
\end{tabular}

Tab. 1. Prüfung der Linearität der Glucose-dehydrogenase-Methode an Hand von 2 Testserien mit je 10 Proben pro Eichpunkt.

\begin{tabular}{|c|c|c|c|c|c|c|c|}
\hline $\begin{array}{l}\text { Glucosese, } \\
\text { [mg/dl] }\end{array}$ & $\begin{array}{l}\text { [mmol/1] } \\
\text { [metzt }\end{array}$ & $\begin{array}{l}\text { Glucose, } \\
\bar{x} \\
\text { [mg/dl] }\end{array}$ & $\begin{array}{l}\mathrm{mt} \\
{[\mathrm{mmol} / \mathrm{l}]}\end{array}$ & $\begin{array}{l} \pm \mathrm{s} \\
{[\mathrm{mg} / \mathrm{dl}]}\end{array}$ & [mmol/1] & $\mathbf{N}$ & $\begin{array}{l}\text { VK } \\
{[\%]}\end{array}$ \\
\hline 500 & 27,5 & $\begin{array}{l}491^{\prime} 80 \\
510^{\prime} 40\end{array}$ & $\begin{array}{l}27,049 \\
28,072\end{array}$ & $\begin{array}{l}6,78 \\
4,20\end{array}$ & $\begin{array}{l}0,373 \\
0,231\end{array}$ & $\begin{array}{l}10 \\
10\end{array}$ & $\begin{array}{l}1,38 \\
0,82\end{array}$ \\
\hline 400 & 22,0 & $\begin{array}{l}397^{\prime} 20 \\
407^{\prime} 50\end{array}$ & $\begin{array}{l}21,846 \\
22,413\end{array}$ & $\begin{array}{l}3,08 \\
2,37\end{array}$ & $\begin{array}{l}0,169 \\
0,130\end{array}$ & $\begin{array}{l}10 \\
10\end{array}$ & $\begin{array}{l}0,78 \\
0,58\end{array}$ \\
\hline 300 & 16,5 & $\begin{array}{l}300^{\prime} 00 \\
310^{\prime} 60\end{array}$ & $\begin{array}{l}16,500 \\
17,083\end{array}$ & $\begin{array}{l}4,42 \\
3,53\end{array}$ & $\begin{array}{l}0,243 \\
0,194\end{array}$ & $\begin{array}{l}10 \\
10\end{array}$ & $\begin{array}{l}1,47 \\
1,14\end{array}$ \\
\hline 200 & 11,0 & $\begin{array}{l}194^{\prime} 90 \\
195^{\prime} 30\end{array}$ & $\begin{array}{l}10,719 \\
10,742\end{array}$ & $\begin{array}{l}1,79 \\
2,41\end{array}$ & $\begin{array}{l}0,098 \\
0,133\end{array}$ & $\begin{array}{l}10 \\
10\end{array}$ & $\begin{array}{l}0,92 \\
1,23\end{array}$ \\
\hline 100 & 5,5 & $\begin{array}{l}101^{\prime} 50 \\
102^{\prime} 40\end{array}$ & $\begin{array}{l}5,583 \\
5,632\end{array}$ & $\begin{array}{l}1,72 \\
1,89\end{array}$ & $\begin{array}{l}0,098 \\
0,104\end{array}$ & $\begin{array}{l}10 \\
10\end{array}$ & $\begin{array}{l}3,51 \\
3,70\end{array}$ \\
\hline 50 & 2,75 & $\begin{array}{l}51^{\prime} 10 \\
51^{\prime} 00\end{array}$ & $\begin{array}{l}2,811 \\
2,805\end{array}$ & $\begin{array}{l}1,79 \\
1,89\end{array}$ & $\begin{array}{l}0,098 \\
0,104\end{array}$ & $\begin{array}{l}10 \\
10\end{array}$ & $\begin{array}{l}3,51 \\
3,70\end{array}$ \\
\hline 25 & 1,375 & $\begin{array}{l}24^{\prime} 30 \\
23^{\prime} 70\end{array}$ & $\begin{array}{l}1,337 \\
1,306\end{array}$ & $\begin{array}{l}1,95 \\
2,58\end{array}$ & $\begin{array}{l}0,107 \\
0,142\end{array}$ & $\begin{array}{l}10 \\
10\end{array}$ & $\begin{array}{r}8,01 \\
10,90\end{array}$ \\
\hline
\end{tabular}




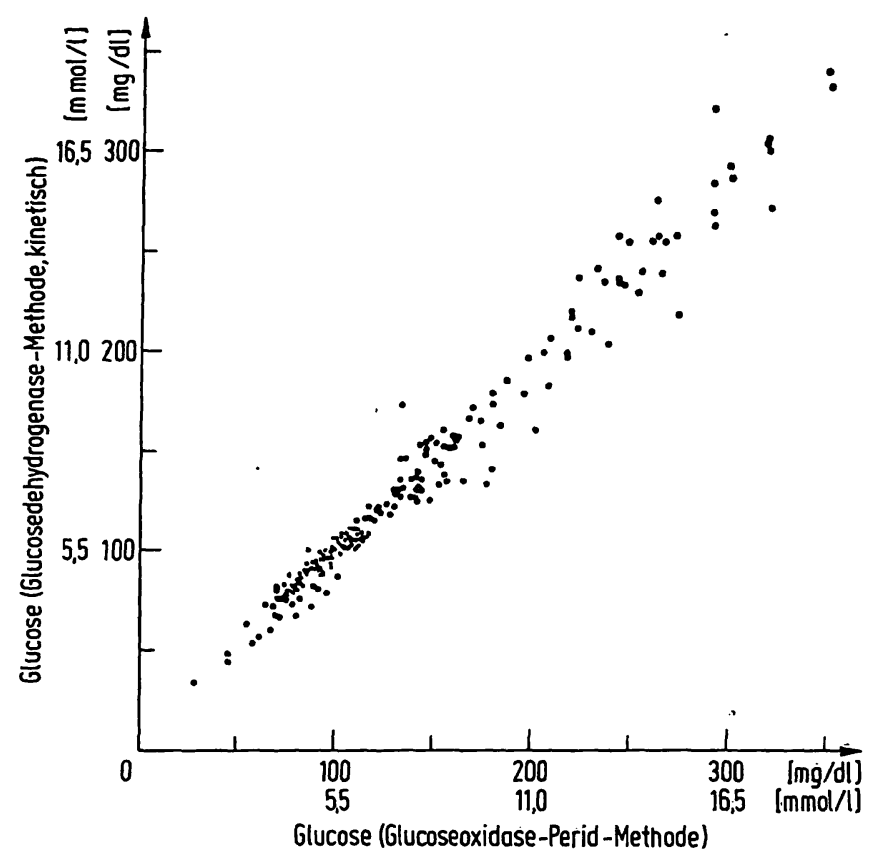

Abb. 2. Korrelation der Meßergebnisse aus der beschriebenen mit der Vergleichs-Methode

\section{Korrelation der Glucosedehydrogenase-Methode mit der Glucoseoxidase-Peridmethode}

Die Abbildung 2 gibt die gefundenen Wertepaare an 200 Patientenproben wieder. Die Berechnung der Korrelation ergibt einen Korrelationskoeffizienten $\mathrm{r}=$ 0,9845 ; die Regressionsgleichung lautet $y=1,065 x$ 4,$312 ; s(x y)=12,374$. Demnach ergibt die Glucoseoxidase-Peridmethode etwas höhere Werte, als sie mit der Glucosedehydrogenase-Methode gefunden werden.

\section{Besprechung der Ergebnisse}

Die vorliegenden Resultate zeigen, daß es möglich ist, mit einem hochauflösenden Photometer und sorgfältiger Temperaturkonstanz enzymkinetische Substratbe- stimmungen durchzuführen, die eine Meßzeit von wenigen Sekunden benötigen. Die hier gewählte Instrumentenkonfiguration ist nicht optimal: Tatsächlich können nicht 12 Glucosebestimmungen in einer̃ Minute durchgeführt werden, sondern die Einzelbestimmung nimmt 15,5 in Anspruch, so daß nur knapp 4 Resultate pro Minute möglich sind. Die konstruktiven Gegebenheiten des LKB-Gerätes erlauben es nicht, den Start der Reaktion, die Reagenzzufuhr, den Mischvorgang und den Vorlauf vor der Meßperiode durchzuführen. Es addieren sich also $5 \mathrm{~s} \mathrm{zu} 5 \mathrm{~s}$ Vorlauf und 5, 5 s Startreagenzzufuhr, Mischvorgang und Probenwechsel. Der theoretisch mögliche Durchsatz von 12 Meßwerten pro Minute wird durch die ungünstigen konstruktiven Gegebenheiten auf weniger aḷ ein Drittel reduziert. Auch die geräteinterne Temperierung der Probenmagazine ist bei der gewählten Durchlaufzeit bei weitèm nicht ausreichend. Der ungünstige Wärmeübergang MetallLuft-Metall-Luft-Kunststoff=wäßrige Lösung macht Aufwärmezeiten von mindestens 15 Minuten notwendig, um von Raumtemperatur auf $35^{\circ} \mathrm{C}$ zu kommen. Darum müssen die vorgefüllten Magazine in einem externen Wärmeschrank auf die Solltemperatur vorgewärmt werden.

Zur Zeit scheint auf dem Instrumentenmarkt kein "schnelles kinetisches Photometer" angeboten zu werden, das ausreichende optische, elektronische und thermomechanische Eigenschạften mit hoher funktioneller Flexibilität verbindet. So lange kein geeigneter Meßplatz zur Verfügung steht, dürfte die Entwicklung „schneller" kinetischer Methoden, d. h. Mesșungen im Bereich von 1-10 Sekunden/Meßansatz stagnieren.

Andererseits würden die Möglichkeiten einer ,Sekundenanalytik" sowohl für die methodologische Weiterentwicklung, als auch im Hinblick auf die Automation Aspekte eröffnen, die dem Ubergang von der Milliliterzu der Mikroliter-Technịk ebenbürtig wären.

\section{Literatur}

1. Keston, A. S. (1956), Abstr. Proc. 129, p 31 C Amer. Chem. Soc. Atlantic City N. J. April 1956.

2. Bergmeyer, H. U. \& Bernt, E. (1974), in Methoden der enzymatischen Analyse (Bergmeyer, H. U. \& Gawehn, M. v. K., eds.) Bd. II, S. 1250-1259, 3. Aufl., Verlag Chemie, Weinheim/Bergstraße.

3. Kadish, A. H., Litle, R. L. \& Sternberg, J. C. (1968), Clin. Chem. 14, 116-131.

4. Hochhorst, H. J., Kreutz, F. H. \& Bücher, T. (1959), Biochem. Z. 332, 18-46.
5. Keller, H. (1971), Med. Laborat. 24-2, 12.

6. Widmer, G. \& Bartels, H. (1972), 8. Internationaler Kongreß Klin. Chem., Kopenhagen 1972

7. Faust, U., Keller, H. \& Becker, J. (1973), Chem. Rundschau 26, 24-29.

8. Banauch, D., Brümmer, W., Ebeling, W., Metz, H., Rindfrey, H., Lang, H., Leybold, K. \& Rick, W. (1975), diese Z. 13, 101-107.
Prof. Dr. H. Keller Institut für Klinische Chemie und Hämatologie des Kantons St. Gallen Frohbergstraße 3 CH-9000 St. Gạllen 